\title{
Numerical Investigation on Aerodynamic Drag and Noise of Pantographs with Modified Structures
}

\author{
Y. Yao ${ }^{1,2}$, Z. Sun ${ }^{2 \dagger}$, G. Li ${ }^{3}$, P. Prapamonthon ${ }^{2,4}$, G. Cheng ${ }^{1,2}$ and G. Yang ${ }^{1,2}$ \\ ${ }^{1}$ School of Engineering Sciences University of Chinese Academy of Sciences, Beijing, 100049, China \\ ${ }^{2}$ Key Laboratory for Mechanics in Fluid Solid Coupling Systems, Institute of Mechanics, Chinese Academy \\ of Sciences, Beijing, 100190, China \\ ${ }^{3}$ CRRC Qingdao Sifang Co., Ltd., Qingdao, Shandong, 266111, China \\ ${ }^{4}$ International Academy of Aviation Industry, King Mongkut's Institute of Technology Ladkrabang, Bangkok, \\ 10520, Thailand
}

†Corresponding Author Email: sunzhenxu@imech.ac.cn

(Received April 2, 2021; accepted August 23, 2021)

\begin{abstract}
It is well known that the pantograph, which works as a complex component of high-speed trains, is an important source of aerodynamic drag and aerodynamic noise of a high-speed train (HST) that can affect HST performance, comfort for passengers, and quietness of nearby communities. Thus, comprehensive studies on aerodynamic characteristics including aerodynamic drag and aerodynamic noise obtained by the pantograph need to be conducted. This work presents the aerodynamic characteristics including aerodynamic resistance and aerodynamic noise generated by the prototype pantograph of a high-speed train running at a speed of 300 $\mathrm{km} / \mathrm{h}$ using numerical techniques of improved delayed detached-eddy simulation (IDDES) and acoustic finite element method (FEM). Then, the structure of the original pantograph is modified by wrapping the insulators, and the base frame, so that aerodynamic resistance and aerodynamic noise may be reduced as expected. Numerical results obtained from the pantograph without modification, with two modifications to the original design i.e. the base frame, and the insulators, are discussed. Compared to the original pantograph, the two modifications of the pantograph at the base frame and the support insulators are conducive to reducing the aerodynamic drag of the pantograph. However, the results also show that the modified insulator may not achieve considerable success in noise reduction. Only the modified base frame shows that noise is reduced significantly. Therefore, this suggests that the pantograph with base frame modification is a better choice for resistance and noise reduction.
\end{abstract}

Keywords: High speed train; Pantograph; Resistance; Aerodynamic noise; Acoustic finite element method.

\section{NOMENCLATURE}

$\begin{array}{ll}\mathrm{C}_{\mathrm{d}, \mathrm{p}} & \text { amplitude of oscillation } \\ \mathrm{C}_{\mathrm{d}, \mathrm{f}} & \text { cylinder diameter } \\ \mathrm{C}_{\mathrm{d}-\mathrm{Total}} & \text { pressure coefficient } \\ L & \text { the distance between the cylinders } \\ D & \text { the diameter of the cylinder } \\ p & \text { the acoustic pressure }\end{array}$

\section{INTRODUCTION}

The reduction of aerodynamic drag and noise is of great significance in the fields of aviation, aerospace, navigation, ground traffic, and so on. Over the last two decades, travel by high-speed trains has played a major role in rapid commute and urban development. Presently, it is a more comfortable way h height

$k \quad$ wave number

$f \quad$ frequency

A amplitude of pressure wave

$\delta t \quad$ time step

$P_{\mathrm{n}} \quad$ the fluctuating pressure of the N-th step for passengers. Nonetheless, as the speed of a highspeed train increases, technical problems in aerodynamic issues such as aerodynamic drag and noise that can be neglected at low speeds become more and more serious. Therefore, the simultaneous reduction of drag and noise with respect to trains operating at speeds over $300 \mathrm{~km} / \mathrm{h}$ should be carefully considered as well. 
It is found that when the speed of a high-speed train reaches $300 \mathrm{~km} / \mathrm{h}$, the aerodynamic resistance accounts for over $75 \%$ of the total resistance (Tian, 2007). Therefore, Sun et al. (2013) performed relevant research on reducing train noise. By studying the distribution characteristics of aerodynamic noise sources around high-speed trains, they studied the different designs of cab window and obstacle remover, and proposed a new design scheme of streamlined head to reduce the noise of the whole vehicle. However, the model is a simplified model without pantograph. For example, an investigation done by Yao et al. (2012) showed that the roof accessories have a significant impact on the aerodynamic performance of the high-speed train. Besides, it was reported in their results that the aerodynamic resistance of the pantograph accounts for about $12 \%$ of the total aerodynamic resistance. In addition, previous studies indicated that running speed plays a major role in aerodynamic noise. For example, a study conducted by Zhang (2009) indicated that aerodynamic noise becomes the main noise source when the train speed exceeds 250-300 $\mathrm{km} / \mathrm{h}$. His results also showed that the aerodynamic noise increases with the sixth power of the train speed. It was also observed by King (1996) that the correlation between the logarithm of the aerodynamic noise and the running speed of the pantograph is approximately linear. Noger et al. (2000) tested a 1/7th scaled train model with and without the pantograph in a wind tunnel. Their results showed that the space near the downstream of the cavity is the most complex and turbulent region, which is the most important noise source of the high-speed train. Investigations from Noger et al. (2000) and Masson et al. (2012) indicated that the pantograph and surrounding shields are the main noise sources of a high-speed train. Liu et al. (2018) used large eddy simulation (LES) together with Lighthill's acoustic analogy to investigate the characteristics of the sound pressure level (SPL), frequency spectrum, and the relation between the SPL and speed of a high-speed train. With the use of a hybrid method of non-linear acoustics solver (NLAS) and Ffowcs Williams-Hawkings (FW-H) acoustic analogy, Yu et al. (2013) studied the aerodynamic noise of the pantograph system, specifically to predict the influence of the pantograph covers on noise within an investigated range of the train speed. Sun and Xiao (2018) and Zhang et al. (2017) used the combined CFD/FW-H with the boundary element method (BEM) to predict aerodynamic noise from the pantograph of a high-speed train. Results obtained by Sun and Xiao (2018) showed the aerodynamic noise from a pantograph, giving the spatial and spectral characteristics of the noise around the pantograph. Results reported by Zhang et al. (2017) indicated that the aerodynamic noise mainly originates from the top regions of the pantograph rather than from the bottom regions. Their results also showed the recognition of aerodynamic noise generated by the pantograph and the largest contribution of pantograph aerodynamic noise is made by several parts of the pantograph, for example, carbon slide plate, balance arm, pantograph head bracket, base frame, insulator.
As reviewed above, although characteristics of aerodynamic drag and noise have been intensively studied, the reduction of drag or noise generated by the pantograph becomes a challenging problem for high-speed-train researchers, designers, and engineers and it needs to be addressed. So far, researchers have tried to find ways to decrease the drag or noise generated by the pantograph. New designs of the geometric shape, passive control methods with different structural materials, and some active control methods have been proposed. Takeshi and $\mathrm{He}$ (2010) used porous materials at the pantograph of a high-speed train. They indicated that the noise is reduced by $1.9 \mathrm{~dB}$ at $360 \mathrm{~km} / \mathrm{h}$ under the wind tunnel test operation. In terms of improving the flow field and noise through geometric shape design, variable cross-section shape and size design were attempted, and bionic surface design such as holes and spiral structures was performed. Takaishi et al. (2004) studied the dipole noise source on the surface of pantograph horns. They analyzed the principle of the noise reduction mechanism of pantograph horns with periodic holes. Suzuki et al. (2008) and Ikeda et al. (2006) optimized the cross-section shape of the pantograph head of a high-speed train. Their results showed that the aerodynamic noise of the pantograph head after optimization is less than that before optimization. By filleting the surface connection on the pantograph base frame and carbon skateboard, Wang et al. (2018) uniformly set small semicircle grooves on a lower surface of a traditional pantograph carbon skateboard in order to alleviate sound production of the pantograph. Their results showed that the bionic optimized pantograph can effectively reduce the amount of vortex shedding, thus reducing the aerodynamic noise. Shi (2018) redesigned the carbon skateboard, balance arm, and insulator of the pantograph by using the elliptical section with the long-short axis ratio of $2: 1$ and shortening the angle size of the pantograph. The four edges of the rectangular bar along the airflow direction of the base frame were rounded. The rest kept unchanged. His results indicated that after optimization, the noise produced by the pantograph head is significantly reduced, and there is no obvious noise reduction effect in the complex parts of the structure. Liu and $\mathrm{Xu}$ (2018) studied the drag reduction and noise reduction of pantograph rod based on bionic non-smooth structure. They showed that the structure of annular corrugated grooves is a good design and can successfully reduce resistance and noise. $\mathrm{Xu}$ et al. (2020) designed the spiral structure of the pantograph rod based on bionics. The results showed that the pantograph rod with a spiral structure has a positive effect on the formation of the cylindrical wake region, which is the main reason for effectively reducing the aerodynamic noise and drag. In the application of the active control method to achieve noise reduction, Takeshi et al. (2016) applied plasma actuators on the surface of the pantograph head. They found that this method can reduce the narrow-band aerodynamic noise generated by the pantograph head. Huang et al. (2019) added a jet to the cavity of the pantograph to reduce the noise actively. With this technique, the sound pressure level (SPL) obtained from monitoring points was reduced by $15.2 \mathrm{~dB}$. Guang et 
al. (2019) studied the aerodynamic noise reduction of the pantograph, including the shape improvement of the pantograph head and bracket, with the Shinkansen pantograph as the investigation model. The application of porous materials and the use of plasma actuator (PA) and synthetic jet actuator (SJA) were proposed.

So far, although there are many studies on the influence of pantograph flow field characteristics, aerodynamic drag, and aerodynamic noise on highspeed trains, most of them focus on drag reduction or noise reduction alone, and few comprehensive studies on drag reduction and noise reduction are presented. In addition, scholars' noise reduction measures for pantographs have the following technical problems. For example, Takehisa et al. (2004) tried to reduce the noise with periodic holes only on the pantograph head. But, in fact, the base and insulator itself are also important noise sources. Moreover, the periodic holes on the pantograph head may have an impact on the strength of the pantograph head, and the pantograph head and the catenary itself have a relatively complex force. So, more practical problems need to be considered in changing its structure. A common defect is that the basic model of the pantograph itself is greatly simplified, and then the geometric design of the insulator or base frame and other components is carried out to explore its drag reduction and noise reduction characteristics. For example, in a study of Shi (2018), the insulator position itself had many spiral coil structures, but the simplified base frame had no spiral coil structure. The structure of the base frame position was also very complex, but the simplified one basically only retained the external rods and intermediate support rods. Therefore, the noise optimization effect obtained by elliptical processing in the insulator position and chamfering in the rectangular edge of the base frame part may be different from that obtained by structural optimization of the actual pantograph model. For the complex original structure of the base frame, only chamfering on the outer edge may almost have no obvious noise reduction effect.

The present work analyzes two design cases that have a good drag reduction effect relative to the original pantograph structure and further analyzes their noise reduction effect. Although the pantograph model has also made some simplification, the degree of simplification is small, basically retaining the structural details of these locations of the base frame insulator. Flow-field physics and sound-field characteristics are comprehensively analyzed using the improved delayed detached eddy simulation (IDDES) and the acoustic finite element method (FEM). Effects of the modification of the pantograph structures on the flow field and noise production are reported. The present paper is divided into five sections, starting with the introduction and numerical algorithms in sections 1 and 2, respectively. Next, computational models, boundary conditions, and grids are presented in section 3. Then, results and discussion are given in section 4. Finally, the conclusion and some remarks are drawn in section 5 .

\section{NUMERICAL ALgORITHMS}

\subsection{Numerical Algorithms for CFD}

In the present work, the numerical approach of improved delayed detached-eddy simulation (IDDES) is utilized to obtain unsteady flow characteristics. In fact, the IDDES was developed from the detached eddy simulation (DES), which was proposed by Spalart et al. (1997). The DES combines large-eddy simulation (LES) and SpalartAllmaras (SA) model The DES can automatically switch between the two models by comparing the local grid scale and wall distance. Spalart et al. (2006) developed the delayed detached-eddy simulation (DDES) by using "Delay LES function" based on Menter's SST model (Menter 1994). The DDES uses the delay function to protect the boundary layer and avoid the mesh-induced separation problem in the original DES, but it leads to a phenomenon of log-layer mismatch (LLM), that is, the vortex viscosity coefficient distribution and velocity type of Reynolds-Averaged Navier-Stokes (RANS) and LES in the logarithmic region does not match well. Shur et al. (2008) proposed the IDDES that can suppress LLM by introducing a lifting function, and introduced a branch of wall modeled LES (WMLES). When the boundary layer grid is sparse, the IDDES can be the DDES, and the IDDES can be automatically switched to the WMLES when the boundary layer grid is dense. This advantage of the IDDES greatly broadens the scope of engineering applications. Based on the IDDES, the definitions of and are the same as DDES, namely,

$$
\begin{aligned}
& L_{R A N S}=\sqrt{k} /\left(C_{\mu} \omega \operatorname{Re}_{\infty}\right) \\
& L_{L E S}=C_{D E S} \Delta
\end{aligned}
$$

But the definition of $\Delta$ needs to be modified according to Eqs. (3) and (4)

$$
\begin{aligned}
& \Delta=\min \left[\max \left(C_{w} d_{w}, C_{w} \Delta_{\max }\right), \Delta_{\max }\right] \\
& \Delta_{\max }=\max \left(\Delta_{i}, \Delta_{j}, \Delta_{k}\right), \Delta_{\text {min }}=\min \left(\Delta_{i}, \Delta_{j}, \Delta_{k}\right)
\end{aligned}
$$

where $C_{w}=0.15$. In the calculation of high Reynolds number problems, the normal grid size near the wall is much smaller than the other two directions. So, the value of near the wall is taken as. With the increase in the wall distance, depending on the characteristics of the boundary layer grid, is taken as or. In both cases, eventually increases to, and the definition of degenerates to the DDES. The IDDES reduces the length scale in the boundary layer, thus reducing the local modular viscosity, which is conducive to the flow instability and turbulence generation in the WMLES branch. The mixed length scale definition of the IDDES introduces the lifting function,

$$
L_{\text {IDDES }}=\tilde{f}_{d}\left(1+f_{e}\right) L_{R A N S}+\left(1-\tilde{f}_{d}\right) L_{L E S}
$$

The main function of is to increase the modular viscosity of the RANS region in the WMLES branch to avoid the LLM phenomenon in the partition of RANS and LES. In fact, for the IDDES based on the SST model, has no obvious effect on the flow (Gritskevich et al. 2012). The term is a delay 
function, which includes the transition function of the DDES branch and the WMLES branch. So, they can be expressed as

$$
\begin{aligned}
& f_{e}=f_{e 2} \cdot \max \left[\left(f_{e 1^{-}}-1\right) \cdot 0\right] \\
& \tilde{f}_{d}=\max \left[\left(1-f_{d t}\right) \cdot f_{B}\right]
\end{aligned}
$$

Other functions are constructed as follows:

$$
\begin{aligned}
& f_{\mathrm{dt}}=1.0-\tanh \left[\left(C_{d t 1} r_{d t}\right)^{C_{d t 21}}\right] \\
& f_{B}=\min \left[2 \exp \left(-9 a_{0}^{2}\right), 1\right] \\
& f_{e 1}=\left\{\begin{array}{c}
2 \exp \left(-11.09 a^{2}\right), a \geq 0 \\
2 \exp \left(-9 a^{2}\right), a<0
\end{array}\right. \\
& f_{e 2}=1-\max \left(f_{t}, f_{l}\right) \\
& f_{\mathrm{t}}=\tanh \left[\left(C_{t}^{2} r_{d t}\right)^{3}\right] \\
& f_{\mathrm{t}}=\tanh \left[\left(C_{l}^{2} r_{d l}\right)^{10}\right]
\end{aligned}
$$

Among them

$$
\begin{aligned}
& r_{d t}=\frac{\mu_{t}}{k^{2} d^{2} \max \left[\left(\sum_{i, j}\left(\partial u_{i} / \partial x_{j}\right)^{2}\right)^{0.5}, 10^{-10}\right]} \\
& r_{d l}=\frac{\mu}{k^{2} d^{2} \max \left[\left(\sum_{i, j}\left(\partial u_{i} / \partial x_{j}\right)^{2}\right)^{0.5}, 10^{-10}\right]} \\
& a=0.25-d_{w} / \Delta_{\max }
\end{aligned}
$$

Here, $C_{d t 1}=8, C_{d t 2}=3, C_{l}=5, C_{t}=1.87$ The function $f_{B}$ is a transition function in the branch of the WMLES, only related to the grid and geometry, which ensures that the thin layer near the wall $\left(f_{B}=\right.$ $1.0)$ is a RANS region, and quickly switches to the LES region $\left(f_{B}=0\right)$ along the normal direction of the wall. When there is no turbulence in the upstream flow, the Reynolds stress is modeled by RANS, and is much greater than 1 , thus $1-f_{d t}>f_{b}$, and $f_{e}=$ 0 . At this time, the length scale of the IDDES becomes

$L_{\text {IDDES }} \approx L_{\text {DDES }}=\left(1-f_{d t}\right) L_{R A N S}+f_{d t} L_{L E S}$

Also, the IDDES reduces the form to the DDES. When the upstream flow has turbulence pulsation (i.e. the upstream flow is analytical), the modulus of the eddy viscosity is small and is far less than $1, r_{d t}$ thus1, 1- $f_{d t}<f_{B}$. Therefore, the length scale of the IDDES becomes

$L_{\text {IDDES }} \approx L_{\text {WMLES }}=f_{B}\left(1+f_{e}\right) L_{\text {RANS }}+\left(1-f_{e}\right) L_{L E S}$

In the RANS to the LES transition region, the IDDES switches to the WMLES branch.

\subsection{Numerical Algorithms for Acoustics}

In the present work, the acoustic finite element method (FEM) is adopted to provide the sound pressure level (SPL), which takes the reflection effect of the structure surface on the noise into consideration. The method of this analysis was also used in the previous work that involved the analysis of aerodynamic noise of the pantograph (YAO et al. 2019).The step of the acoustic FEM starts from calculating the noise propagation from the Helmholtz equation as defined in Eq. (19)

$$
\bar{\nabla}^{2} \cdot p(x, y, z)+k^{2} p(x, y, z)=\mathrm{f}(x, y, z)
$$

Where $p$ is the acoustic pressure, $k$ is the wave number, $f$ is the frequency, the corresponding wavelength is computed from Eq. (20)

$$
\lambda=2 \pi / k=2 \pi c / \omega=c / f
$$

Then, the method of the fast Fourier transform (FFT) is adopted for spectral analysis. The square of the amplitude of pressure wave is expressed in terms of the sum of sine and cosine functions, as seen in Eq. (21).

$$
\begin{aligned}
& A^{2}\left(\omega_{k}\right)=2\left[\frac{1}{N} \sum_{n=1}^{N-1}\left(p_{n}-\bar{p}\right) \cos \left[\frac{2 \pi n k}{N}\right]\right]^{2} \\
& +2\left[\frac{1}{N} \sum_{n=1}^{N-1}\left(p_{n}-\bar{p}\right) \sin \left[\frac{2 \pi n k}{N}\right]\right]^{2}
\end{aligned}
$$

Where $P_{n}$ is a data set and it represents the fluctuating pressure of the $\mathrm{N}$-th step; $\mathrm{n}=0,1,2, \ldots$, $\mathrm{N}-1$. Meanwhile, $\omega_{k}=k / N \delta t$ here $\delta t$ is the time step. Finally, the SPL is computed by Eq. (22).

$\operatorname{SPL}\left(\omega_{k}\right)=10 \log \left(A^{2} / P_{\text {ref }}^{2}\right)$

where $P_{\text {ref }}$ is constant and equal to $2 \times 10^{-5} \mathrm{~Pa}$.

\subsection{Numerical Algorithms for Acoustics}

This section presents the validation of computational methods i.e. the IDDES and FEM. Since many structures of the pantograph could be treated as cylinders, the flow field of the flow past tandem cylinders is used for the IDDES and FEM validations. Figure 1(a) displays the computational domain used for the validation. Each cylinder has a diameter $(D)$ of $0.05715 \mathrm{~m}$ and a length of $3 D$. The distance between the cylinders $(L)$ is 3.7D. Figure 1 (b) shows the computational grid generated for the validation. The trimmer meshing method is employed and the first-layer thickness is $0.001 \mathrm{~mm}$ with a growth ratio of 1.1 in the wall-normal direction. The grid size of the cylindrical surface is 1 $\mathrm{mm}$. The total elements of the computational grid are about 8.5 million. For boundary conditions, the pressure outlet is set as gauge pressure of $0 \mathrm{~Pa}$, whereas the inlet condition is set as a uniform velocity of $44 \mathrm{~m} / \mathrm{s}$. the other parallel planes are given as symmetry. The IDDES is used to calculate the flow field. The pressure-velocity coupling method is addressed by the semi-implicit method for pressure linked equations (SIMPLE) algorithm. The discrete algebraic equation is solved by the Gauss-Seidel iteration technique.

Figure 2(a) shows the generation, development, and detachment of the leeward vortices downstream of the two cylinders using instantaneous iso-surface normalized Q-criterion at $\mathrm{Q}=10000$. The vortices alternate axially in the downstream direction of the flow. Clearly, the leeward side vortices are mainly 
generated when the airflow flowing from the cylindrical wall surface leaves the leeward sidewall surface. The generation, development, and shedding of the vortices lead to noise generation. In the present work, the mean square root of the pressure coefficient $\left(C p_{\text {rms }}\right)$ of the downstream cylinder surface (measured from upstream, clockwise) and

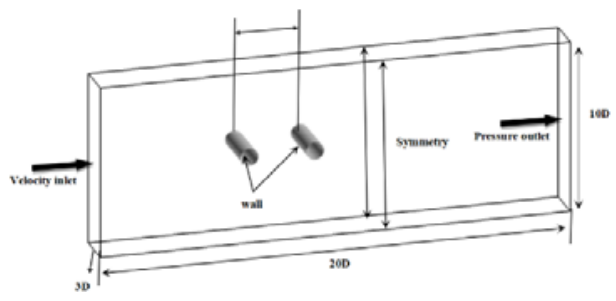

(a)
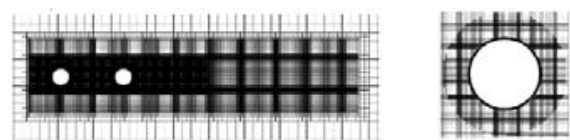

(b)

Fig. 1. (a) Geometry and computational domain and (b) computational grid for flow field.
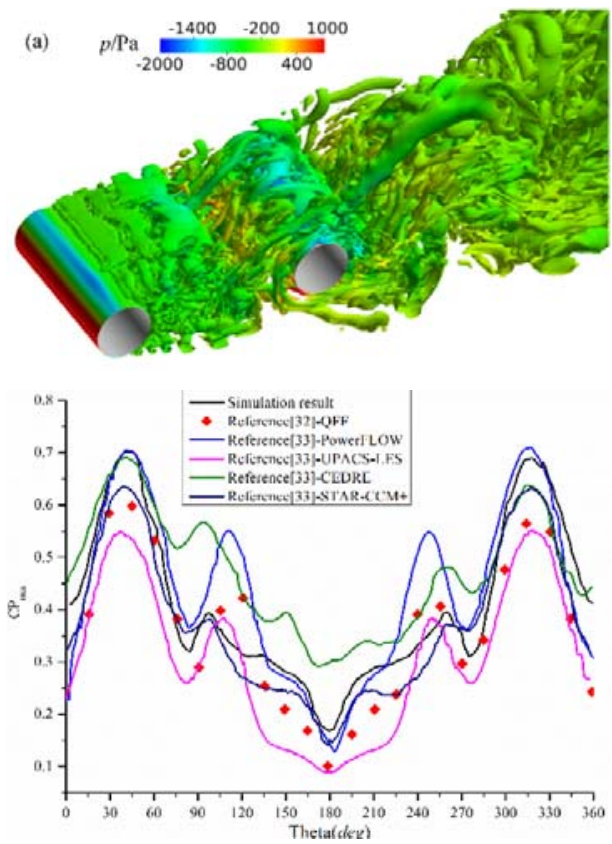

(b)

Fig. 2. (a) Instantaneous iso-surface normalized $\mathrm{Q}$-criterion $(\mathrm{Q}=\mathbf{1 0 0 0 0})$ and $(\mathrm{b})$ root-mean-square pressure coefficient on cylinder downstream surface.

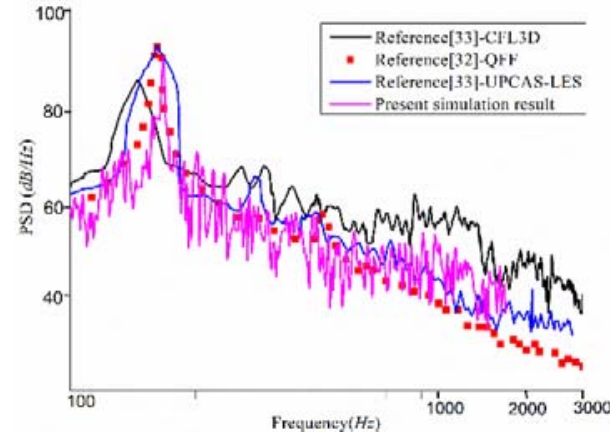

Fig. 3. Results of noise located at (9.11D, 32.49D).

the noise at a position of $(9.11 D, 32.49 D)$ in the $\mathrm{X}-\mathrm{Y}$ plane with the first cylinder center as the coordinate reference origin are selected. The results are compared with those reported by Langley Research Center of NASA (Neuhart et al. 2009) and some selected results in the literature (Lockard 2011). As shown in Fig. 2(b), when $=45$ on the downstream cylinder surface, $C p_{\text {rms }}$ can be observed and the results basically meet the calculation accuracy. After validating $C p_{\text {rms }}$, results obtained by the acoustic FEM are validated, as shown in Fig. 3. Specifically, the noise at the far-field monitoring points $(9.11 \mathrm{D}$, 32.49D) shows that the FEM is effective and acceptable for more complicated calculations of the noise caused by the double cylinder disturbance.

\section{COMPUTATIONAL MODELS, BOUNDARY CONDITIONS AND MESH}

\subsection{Computational Models}

To study aerodynamic characteristics including aerodynamic drag and noise, at first, the geometry of the original pantograph structure is used, as shown in Fig. 4(a). Then, to improve aerodynamic characteristics and to alleviate the negative effects of aerodynamic drag and noise, the original pantograph is modified by wrapping the insulators and base frame, as shown in Figs. 4(b) and (c), respectively. For the treatment of the insulators in Case 2, the design of elliptical structure wrapping is adopted. The short axis is perpendicular to the flow direction and the long axis is parallel to the flow direction. The ratio of the short axis to the long axis is 1:1.5. For the treatment of the base frame part in Case 3, the complex structures of different scales on the base frame are wrapped directly by a large-scale smooth surface and chamfered on the edge corners. To be convenient for mention these cases, from now onwards, Cases 1, 2, and 3 are used for the original structure, the modification of the structure with wrapping the insulators, and the modification of the structure with wrapping the pantograph base frame, respectively. 


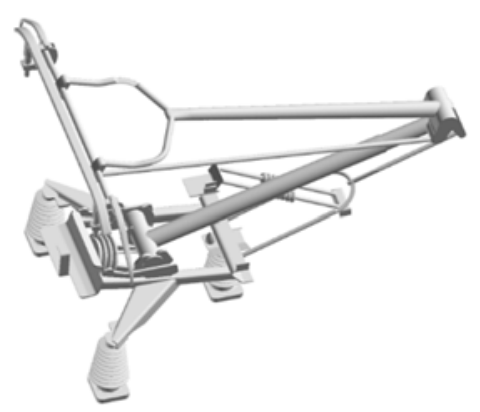

(a) Case 1

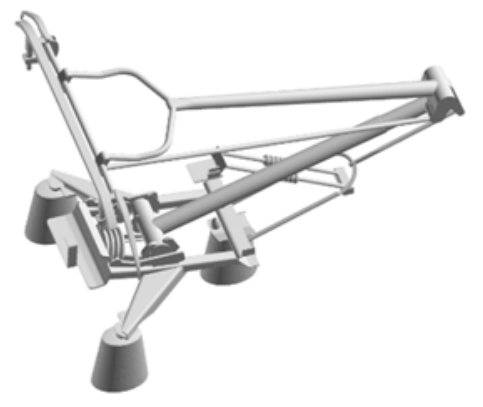

(b) Case 2

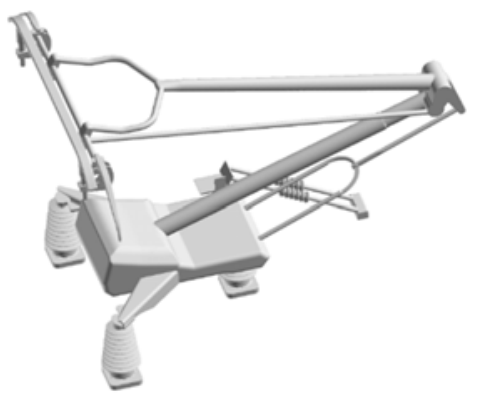

(c) Case 3

Fig. 4. Three types of the pantograph models: (a) Case 1: Original structure, (b) Case 2: Wrapping insulator, and (c) Case 3: Wrapping base frame.

\subsection{Boundary Conditions and Meshing Strategy}

The computation domain with a size of $10 H \times 5 H \times$ $2.5 H$, where $H$ is the height of the pantograph and $H$ $=1.6 \mathrm{~m}$, is depicted in Fig. 5. The STAR-CCM+, a commercial software, is used to generate Cartesian grids. According to the grid arrangement of the flowfield region, the thickness of the first layer near the wall is $0.05 \mathrm{~mm}$ with a growth rate of 1.2 . To resolve flow in the boundary layer, the number of the grid layers close to the train surface is 10 . The grid size on the pantograph surface is $10 \mathrm{~mm}$, and the mesh refinement is employed in the area near the train body, in the rear of the train body, and the region around the pantograph so that phenomena of the flow physics can be captured. Additionally, the isotropic mesh is used in the whole computational domain except the boundary region. Figure 6 shows grids around pantograph surfaces and it can be seen that grids in the region near the pantograph are refined. The total number of the computational grids is about 30 million elements. In flow field analysis, the incoming velocity is set at $300 \mathrm{~km} / \mathrm{h}$. The Reynolds number, Re, is $8.9 \times 10^{6}$ based on the incoming velocity and the height $\mathrm{H}$ of the train. The boundary conditions are set as follows: (1) The surface of the train and the ground are fixed walls with non-slip and non-penetration conditions. (2) The other boundaries are given as free-stream conditions. For the meshindependence study, it was given in the previous work (YAO et al. 2019).

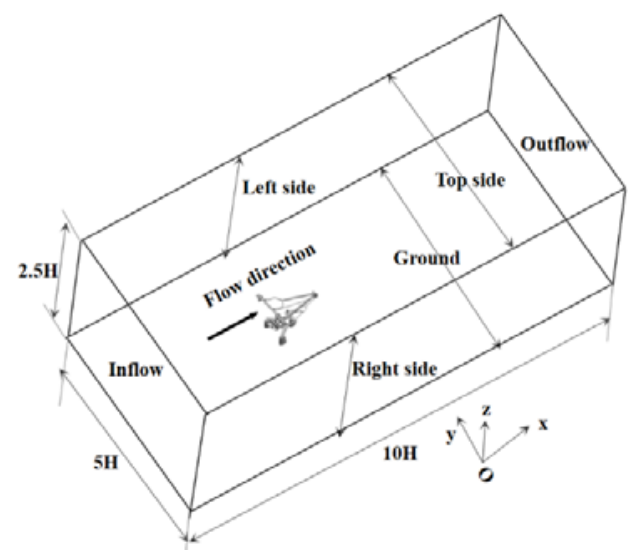

Fig. 5. Computational domain.

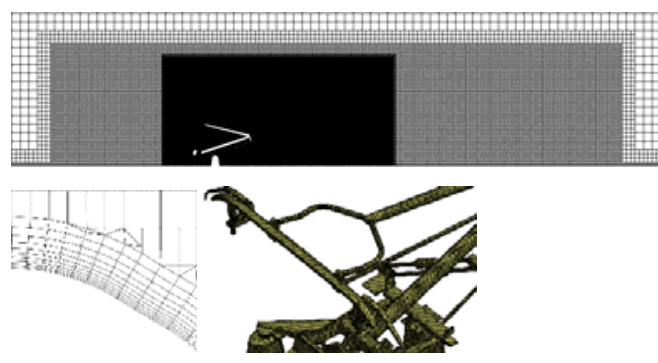

Fig.6. Distribution of computational mesh including volume mesh near the surface; and surface mesh of the pantograph.

\section{RESULTS AND DISCUSSION}

\subsection{Instantaneous Flow Structures and Aerodynamic Force}

As previously mentioned in the introduction part that the purpose of this work is to predict the effects of different structures of the pantograph on drag reduction and noise reduction, the calculation efficiency is improved by only considering the pantograph body itself in the analysis. The original structure of the pantograph is shown in Fig. 7(a). To understand the influence of different parts of the pantograph on overall resistance and noise, the pantograph is divided into six parts i.e. 1. pantograph head, 2. upper arm, 3. corner, 4. lower arm, 5. base 
frame, and 6. insulators. And the contribution of each component to the resistance is shown in Fig.7(b). The pressure distribution on surfaces of the pantograph structure and the surrounding flow field is the main reason for aerodynamic loads.

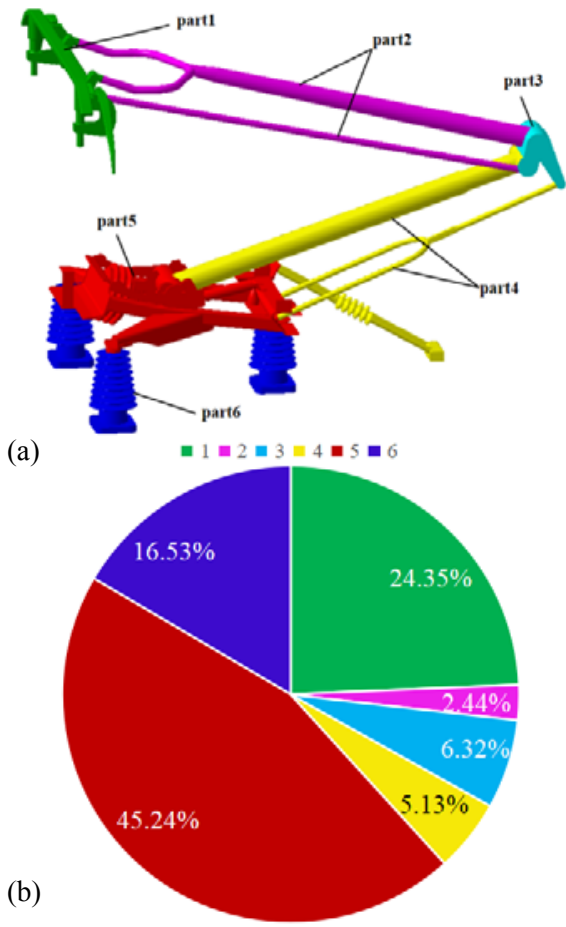

Fig.7. (a) Original pantograph model; (b) Resistance obtained by each part of pantograph in original case. 1: Pantograph head, 2: Upper arm, 3: Corner, 4: Lower arm, 5: Base frame, and 6: Insulators.

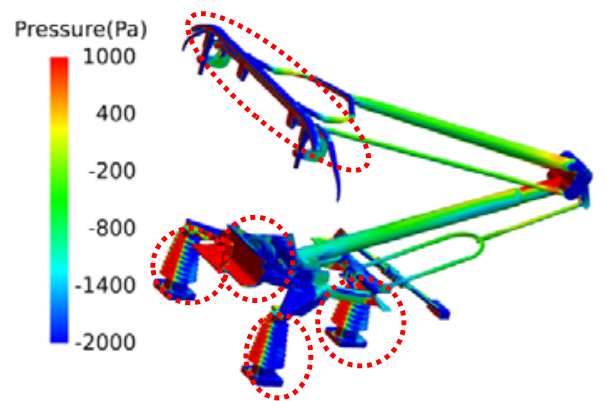

Fig. 8. Surface pressure distribution of original pantograph.

As shown in Fig. 8, the surface pressure distribution is given first. One can observe that the higher amplitudes of the pressure are found in the windward regions of the pantograph i.e., pantograph head, base frame, upper and lower arms connecting corner, and insulators. This is consistent with the analysis of the contribution of each component to the resistance in Fig. 6(b). It can be observed that the base frame, pantograph head, and insulators account for a large proportion of the resistance. Besides, Fig. 6(b) also indicates that the most influential component is the base frame, followed by the pantograph head, and insulators, respectively.

For each component of the pantograph, the flow characteristics around the pantograph and drag reduction are taken as the main objective to modify the pantograph shape. Also, the influence of aerodynamic noise and its propagation to the farfield are considered. It should be noted that due to the complex connection between the pantograph head and catenary, the design of the pantograph head is ignored in this work. Only the modification of the base frame and insulators are considered as they contribute greatly to the resistance. As previously mentioned in section 3.1, the first and second modifications are done by wrapping the insulators and base frame, as early shown in Figs. 4(b) and (c), respectively. To obtain a better understanding of the characteristics of flow and aerodynamic noise source, as shown in Fig. 9, the structure of the surrounding flow field obtained by the three shapes of the pantograph is analyzed by the instantaneous iso-surface pressure normalized by $\mathrm{Q}$-criterion $(\mathrm{Q}=$ 20000). It is found that different structures induce different scales of the vortex and the strong interference between vortices induces many complex structures of the vortex behind the pantograph. In general, the pantograph wake area has the following flow characteristics:

(a) The downstream vortices of the component fall off and interfere with each other, forming hairpin vortices of different sizes.

(b) Strong interference generates strong turbulence, forming significant aerodynamic noise sources. The vortices produced by each part of the pantograph interact with each other at the rear, forming complex wake structures. The aerodynamic force changes, at the same time, the interaction of vortex generation and shedding changes the pressure on the surface of the pantograph and produces fluctuating pressure, which induces the aerodynamic noise of the pantograph and forms different noise results.

However, there are also differences in the vortices behind the three different structures. For the original case, Case 1, there are a large number of complex vortices behind the bow. In Case 3, the scale of the base frame becomes uniform after wrapping the base frame with a complex structure and large size differences. The vortex formed after the airflow flows past the large-scale base frame is simplified, which has a significant impact on the flow field. At $\mathrm{Q}=20000$, it can be observed that the vorticity in the tail space region of Case 3 is obviously smaller than that of Case 1. For Case 2, there is no obvious change in the vorticity in the space. But compared to Case 1 with more spiral coil structures at the insulators, after elliptical wrapping in Case 2, the vorticity after the insulators is slightly reduced.

To clearly understand the flow field behind the pantograph, a cross-sectional area at $\mathrm{y}=0$ is provided for a detailed analysis of the vorticity, as displayed in Fig. 10. One can see that in the rear of 


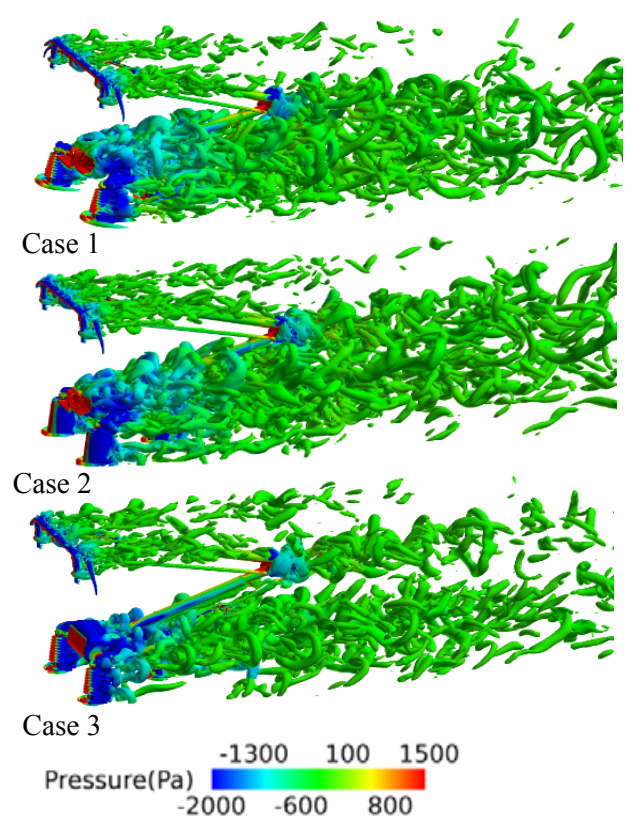

Fig. 9. Instantaneous iso-surface pressure normalized by $Q$-criterion $(Q=20000)$. Case 1 : original structure, Case 2: wrapping insulators, and Case 3: wrapping base frame.
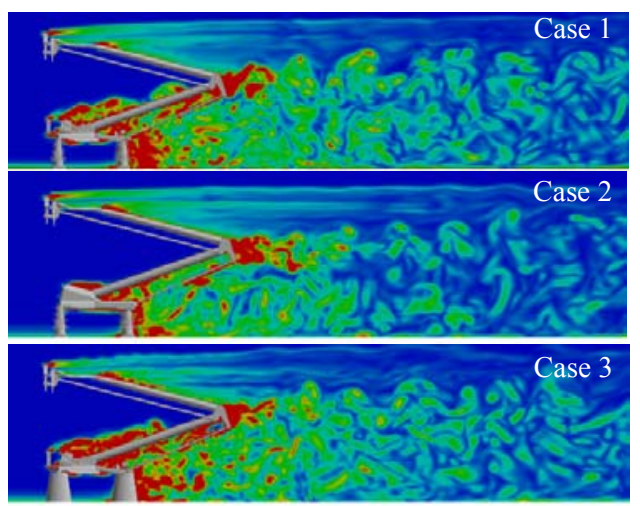

Vorticity Mag(1/s) $\begin{array}{cccc}200 & 600 & 1000 \\ 0 & 400 \quad 800\end{array}$

Fig. 10. Vorticity magnitude $(1 / \mathrm{s})$ on plane at $y=$ 0 obtained by Case 1: original structure, Case 2: wrapping insulators, and Case 3: wrapping base frame.

the original pantograph structure (Case 1), it is easy to produce flow separation and a large number of horseshoe vortices appear, forming a complex phenomenon of tail vortex shedding, especially behind the base frame and insulator structures. Another observation is that the wake vortices are dense and move backward alternatively. These phenomena are considered as a source of the greater resistance of the pantograph structure. Compared with the original shape, the vorticity around the upper arm and the lower arm increases after the insulators are wrapped (Case 2), but the vorticity behind the wrapped insulators is weakened. This may be explained by the fact that when the insulators are wrapped, the fluid flows backward from the covered surface to avoid passing through a large number of coils on the insulator surfaces, and then the alternating horseshoe vorticity behind the insulators decreases slightly. For Case 3, when the base frame is wrapped, the vorticity attenuation of the base frame and the latter part is obvious, the vorticity energy behind the base frame and insulators is obviously weakened compared with Case 1. From the instantaneous iso-surface pressure normalized by Q-criterion and the vorticity on the plane at $y=0$, it predicts that the airflow pulsation produced by Case 3 is weaker and the noise reduction effect is better.

The results of the pantograph resistance in terms of $C d, p, C d$,f, and $C d$-Total obtained by the three shapes are listed in Table 1. It should be noted that $\mathrm{Cd}, \mathrm{p}$ is the pressure drag coefficient that evaluates the pressure resistance, whereas $\mathrm{Cd}, \mathrm{f}$ is the frictional drag coefficient that describes the frictional resistance. Cd,Total represents the sum of $\mathrm{Cd}, \mathrm{p}$ and $\mathrm{Cd}$,f. The running speed of the train for all cases is $300 \mathrm{~km} / \mathrm{h}$ (about $83.333 \mathrm{~m} / \mathrm{s}$ ), and the reference area is $1 \mathrm{~m}^{2}$ for comparison.

Table 1 Cd,p, Cd,f, Cd,Total, and drag reduction obtained by different pantograph structures

\begin{tabular}{|c|c|c|c|c|}
\hline $\begin{array}{c}\text { outlin } \\
\mathrm{e}\end{array}$ & $C d_{, p}$ & $C d_{, f}$ & $\begin{array}{c}C d- \\
\text { Total }\end{array}$ & $\begin{array}{c}\text { Percentag } \\
\text { e change }\end{array}$ \\
\hline Case 1 & $\begin{array}{c}0.429 \\
6\end{array}$ & $\begin{array}{c}0.012 \\
3\end{array}$ & $\begin{array}{c}0.441 \\
9\end{array}$ & $/$ \\
\hline Case 2 & $\begin{array}{c}0.412 \\
9\end{array}$ & $\begin{array}{c}0.011 \\
4\end{array}$ & $\begin{array}{c}0.424 \\
3\end{array}$ & $-3.98 \%$ \\
\hline Case 3 & $\begin{array}{c}0.353 \\
7\end{array}$ & $\begin{array}{c}0.014 \\
3\end{array}$ & $\begin{array}{c}0.368 \\
0\end{array}$ & $-16.71 \%$ \\
\hline
\end{tabular}

It can be seen that the trends of $C d_{, p}$ and $C d$-Total are the same, namely, the values of Case $1>$ Case $2>$ Case 3. However, $C d_{\text {,f }}$ of Case 3 is maximum, whereas $C d_{\text {,f }}$ of Case 2 is minimum. These results imply that both modified shapes, Cases 2 and 3, are conducive to the reduction of pantograph resistance, especially Case 3, namely, when the base frame is wrapped. For the modified insulators, due to the complex shape of the insulators, the wrapping of the insulators can prevent incoming flow through the insulator coils, reduce the contact area, and reduce the frictional resistance slightly. Furthermore, with the oval covering in a truncated cone of the insulators, the windward face is the face corresponding to the short axis, and the smooth surface is conducive to the air flow backward. This causes the pressure resistance to be weakened, thereby reducing overall resistance.

However, the friction resistance will increase when the base is covered, because the large area of complex structure increases the contact area with the airflow. This phenomenon is opposite to the pressure resistance because the surface of the original base frame is concave. Therefore, the incoming flow could not flow backward quickly, resulting in a large inviscid resistance. But, when the base frame surface 


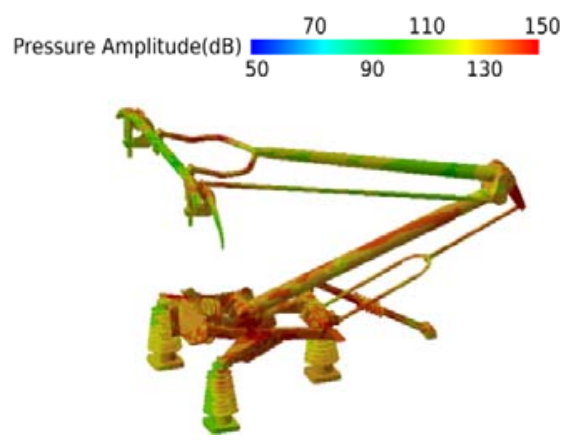

Case1

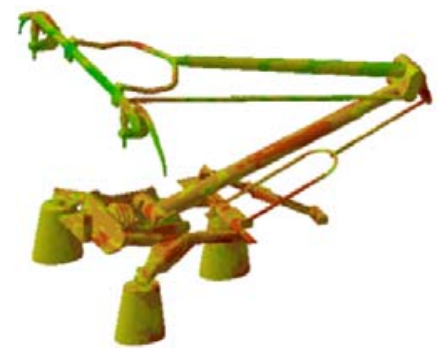

Case2

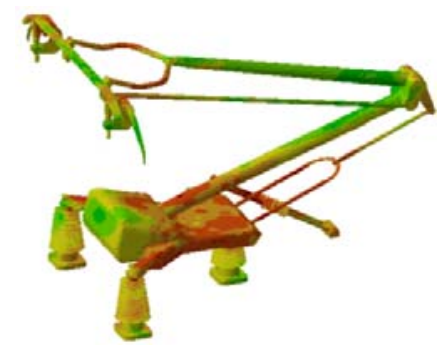

Case 3

$f=200 \mathrm{~Hz}$

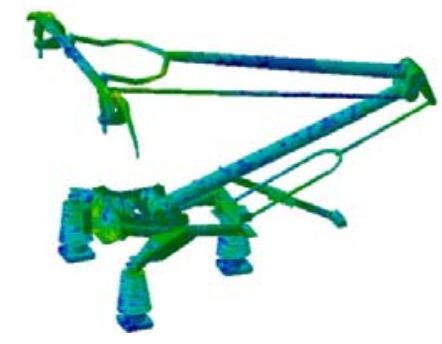

Case1

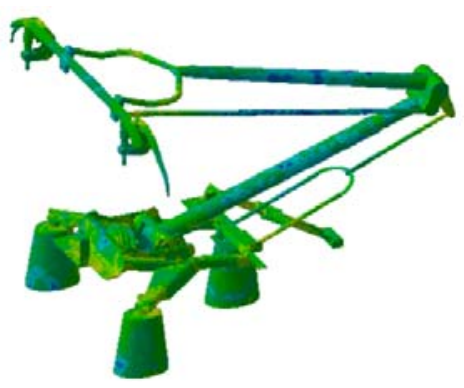

Case2

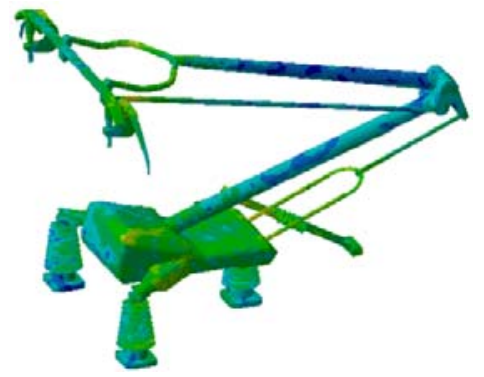

Case3

$f=1000 \mathrm{~Hz}$

Fig.11. Aerodynamic pressure fluctuation amplitude at 200 and $1000 \mathrm{~Hz}$ for Case 1: original structure, Case 2: wrapping insulators, and Case 3: wrapping base frame.

becomes convex, the incoming flow can flow backward quickly, and the flow is faster after the rear part is wrapped. Consequently, the inviscid resistance significantly reduces and the total resistance decreases. The influence of shape change on noise will be discussed in the next section.

\subsection{Aerodynamic Noise Source}

This section presents a comparison of the aerodynamic noise source and its near fields obtained by the three cases. Figure 11 indicates the presence of the surface sound source by evaluating soundlevel distributions in terms of the pressure amplitude occurring on the surface at the frequencies of $200 \mathrm{~Hz}$ and $1000 \mathrm{~Hz}$ for the three cases. Obviously, along the direction of the incoming flow, the sound source at the upstream of the pantograph is weak, whereas the sound source at the downstream is strong. This is because the upstream, especially at the leading edge, is always collided with the incoming flow rapidly, forming an upwind stagnation area. Therefore, the pressure fluctuation is small. This phenomenon is different from the downstream, namely, as the detached vortices of the upstream moving backward collide with the downstream components, causing the sharp change of the pressure fluctuation on the downstream components. Thus, the amplitude of the sound source is higher at the back of the base frame, lower arm, and insulators. The amplitude of the sound source on the surface decreases with increasing frequency, too.

In addition, compared to the original pantograph for both frequencies, in general, the pressure amplitude of the surface sound source in Case 3 is significantly improved. As previously seen in section 4.1 that the base frame is a very important sound source, it affects the flow field greatly. The flow field is also disturbed by the lower arm rod because it is directly connected with the base frame. However, after wrapping the base frame, the pressure amplitudes of the sound source of the base-frame surface and the upper part of the lower arm rod and the corner are reduced. Regions with relatively high amplitudes are observed at $f=200 \mathrm{~Hz}$. It should be noted that the amplitude distribution of the surface sound source 
can only qualitatively judge the possible noise radiation effect from each shape. The larger the amplitude is, the more intense the surface unsteady flow is, which may induce higher noise. However, since the noise radiation can provide a directional effect and the noise radiation is greatly affected by the environment, the noise characteristics at different positions need to be analyzed in detail. To analyze the pressure fluctuation on the surface the pantograph, seven probes on the surface of the pantograph are monitored i.e. three probes on the head, one probe behind the base frame, one probe at the upper and lower arms, and one probe at the corner, as illustrated in Fig. 12. The FFT is used to calculate the spectrum of the sound pressure level (SPL) at the seven monitoring positions.
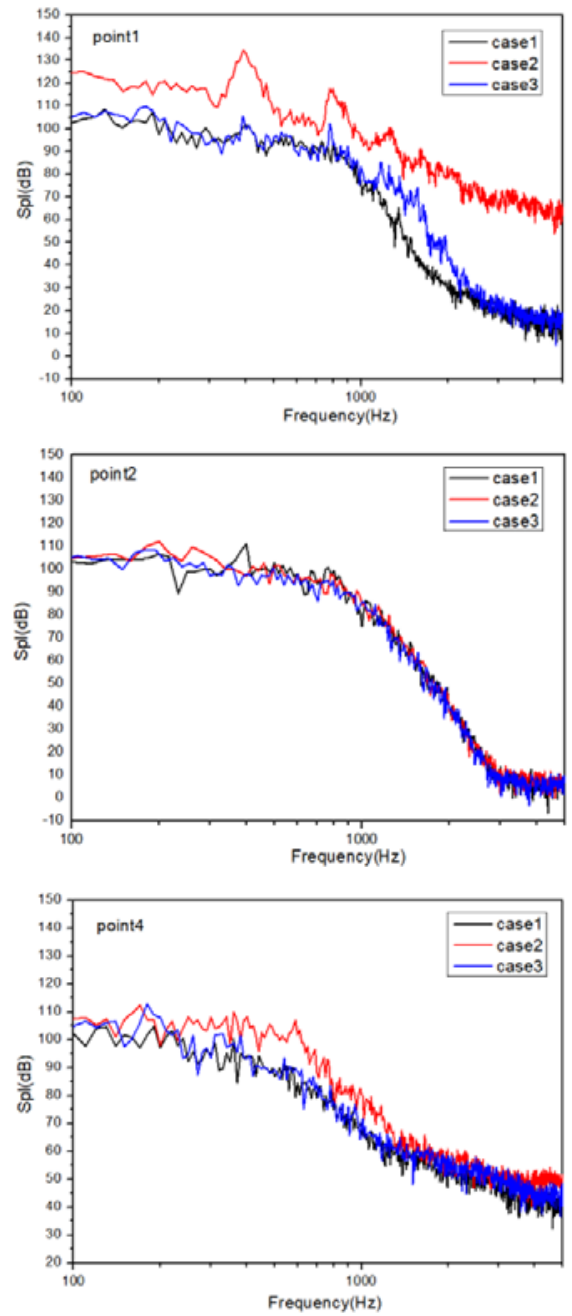

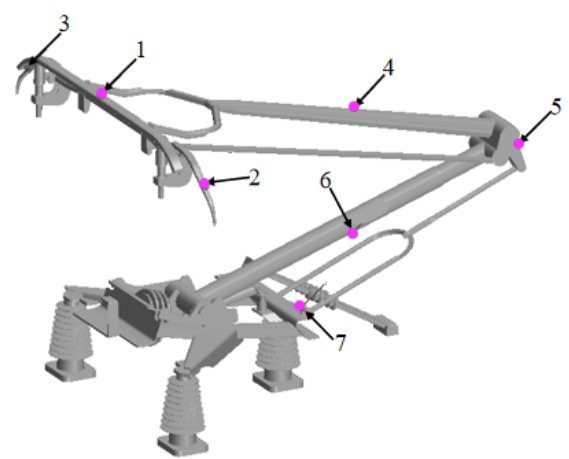

Fig. 12. Positions for monitoring pressure fluctuation on the surface of pantograph.
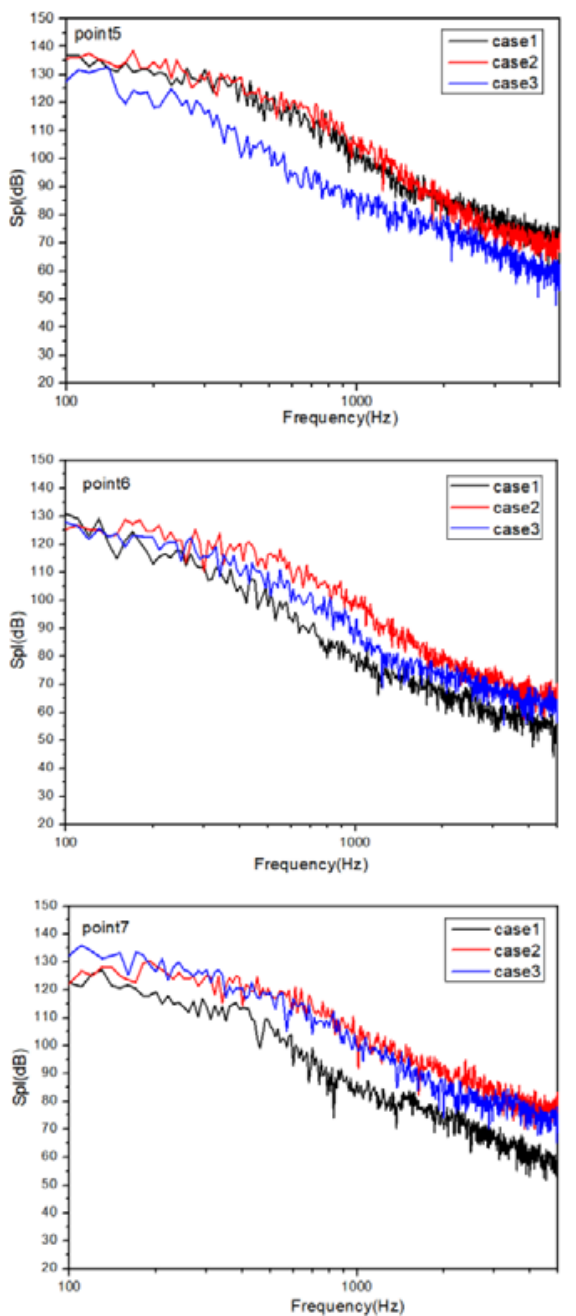

Fig. 13. SPL obtained by seven monitoring positions on the surface of pantograph for Case 1: original structure, Case 2: wrapping insulators, and Case 3: wrapping base frame.

As presented in Fig. 13, the sound amplitudes at all monitoring positions decrease with the increase in the frequency. Relatively large fluctuations of the SPL are found at positions 5, 6, and 7 where are behind the corner, lower arm, and behind the base frame, respectively. Specifically, when considering the whole range of the frequency, Case 2 provides higher SPLs for these monitoring positions. The reason is that positions 5,6 , and 7 are located behind the complex parts, where their flow fields are quite 
complex. This causes large pressure fluctuations, and disturbs airflow, thereby inducing aerodynamic noise. As a result, the SPL increases at such positions. For Case 3, it is clear that the smallest overall amplitude is found at position 5 where is located behind the corner. This is mainly because of the fluctuation of the flow field caused by the wrapping base frame. At position 7, the difference between the overall amplitudes obtained by Cases 3and 2 is small, and the overall amplitudes obtained by Cases 3 and 2 are larger than those by the original case. Other interesting phenomena are observed as well. At position 1, the noise induced by Case 2 has the highest SPL values in the whole frequency range. It implies that the presence of the wrapping at the insulators leads to the highest SPLs at the head of the pantograph. Furthermore, with wrapping the insulators, it has a great impact on the space flow field around the whole pantograph, which can change the pressure fluctuation of the entire pantograph. The overall influence of the shape changes of the insulators on the flow field around the upper part of the pantograph is more obvious than that of the shape change of the base frame. It may be because the position of the insulators is close to the vehicle surface. In this work, the position of the insulators is close to the bottom surface, which can result in a complicated flow field in the boundary layer at the bottom surface. Moreover, the insulators originally consist of multi-layer coils in an elliptical shape. The bottom airflow can pass through the insulators better and faster, and the corresponding airflow from the top to the rear may be filled at the bottom. As a result, it greatly affects the flow field around the upper component, thereby causing the pressure fluctuation on the surface of each component. For Case 3, since the base frame is located at the top of the bottom of the pantograph and the original base frame has several complex structures with different sizes, it is expected that the flow field around this part is complicated. After the base frame is wrapped, a smooth surface is formed and it drives a large amount of surrounding airflow to flow backward rapidly. Therefore, the influence on the flow field in the upper and lower regions and even the whole space is more obvious. However, it should be noted that the influence of the shape modification at any position on the whole spatial flow field is complex. This is related to the position of the specific bow component and the geometric shape of the corresponding component. For the remainder of the positions, at position 2, it is located at the pantograph angle. The modification of the shape of the base frame and insulators has a slight effect on the flow field in this region, thus obtaining insignificant differences in the SPL for the three cases. At position 4, in general, Case 2 gives the highest SPL amplitudes.

\subsection{Aerodynamic Noise Propagation and Attenuation Characteristics}

This section presents results obtained by monitoring positions in the far-field regions where are perpendicular to the side of the pantograph body along the y direction away from the reference point as shown in Fig.14. The reference point is set at the middle position of the $\mathrm{x}$-direction coordinates before and after Case 1 base frame, and $z=0.5 \mathrm{~m}$ away from the bottom of pantograph.

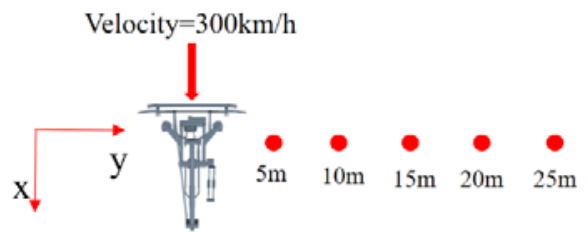

Fig. 14. Monitoring positions in far-field region of pantograph.

Figures 15(a)-(d) shows comparisons of the noise spectrum characteristics at $25 \mathrm{~m}$ monitoring positions when the representative pantograph head (part 1), corner (part 3), base frame (part 5), and insulator (part 6) are selected as noise sources, respectively. Clearly, the spectrum characteristics of the far-field noise induced by different components change when the pantograph is modified.

Figure 15(a), which shows the noise induced by the pantograph head, shows that the main frequency is between $400 \mathrm{~Hz}$ and $500 \mathrm{~Hz}$, but the amplitudes corresponding to the main frequency decrease after the shape of the insulators or base frame is wrapped. Figure 15(b), which is the noise induced by the corner part of the connecting upper and lower arms, indicates that the noise induced by Case 3 is the smallest among the three cases. This reflects the influence of the shape change of the base frame on the whole spatial flow field. As the corner is located at the rearmost part of the whole pantograph, the spectrum curves of far-field noise induced by this part also indicate that the other two cases induce larger noise, which may be predicted to have a great contribution to the far-field noise induced by the whole pantograph. Figure 15(c) presents the noise caused by the base frame. It is found that the noise induced by the base frame increases when Case 2 is considered. This phenomenon is different from Case 3 , namely, the noise caused by the base frame of Case 3 is close to that of Case 1. Figure 15(d) shows the noise induced by the insulator part. One can see that the noise caused by the insulator in Case 2 is significantly higher than that in Case 1, and the noise caused by the insulator in Case 3 is also slightly larger than that in Case 1. These phenomena may be explained by the fact that as Case 2 is the wrapping insulator part, it results in a large change in the surrounding flow field. As a result, it causes the variation of the surface pressure fluctuation and the results of the far-field noise reflect this variation. For Case 3, the large-area wrapping of the base frame not only changes the flow field around the base frame but also the whole spatial flow field, including the flow field around the insulators directly connected to the base frame. This further affects the far-field noise characteristics.

In general, the shape modification of the pantograph affects not only local flow fields in modified areas but also disturbs the whole spatial flow field. 
(a)
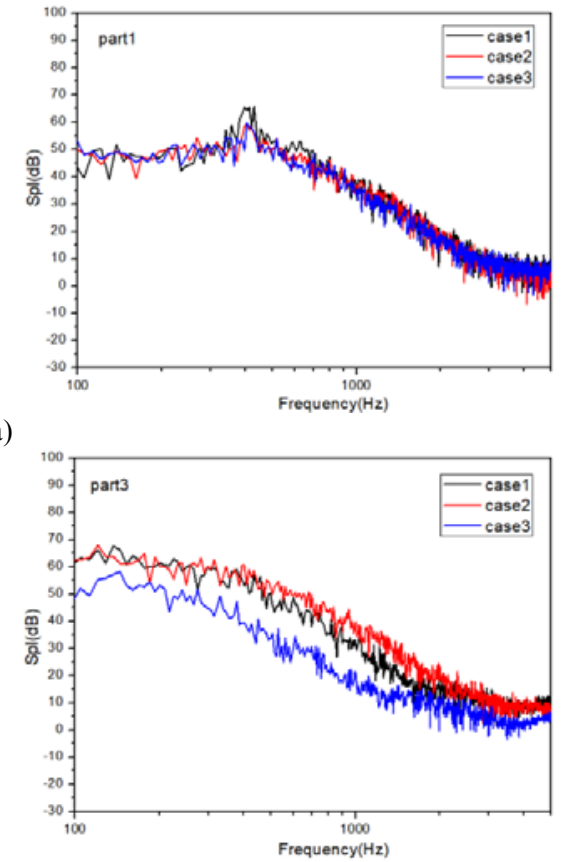

(b)

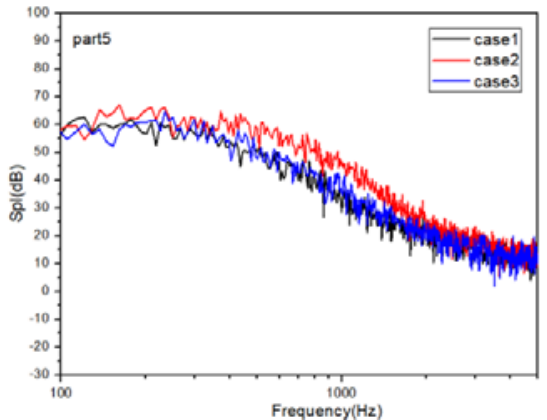

(c)

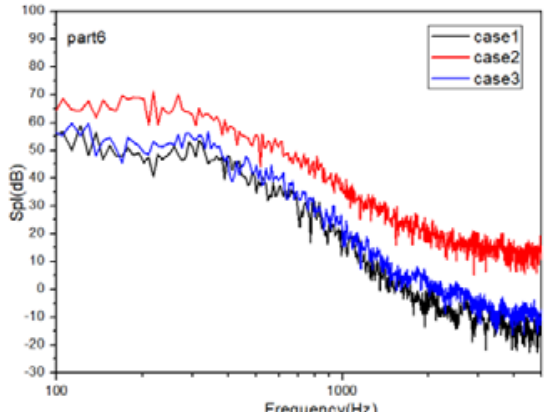

(d)

Fig. 15. Noise spectrum characteristics at $25-\mathrm{m}$ monitoring positions induced by (a) pantograph head (part 1), (b) corner (part 3), (c) base frame (part 5), and (d) insulator (part 6) for Case 1: original structure, Case 2: wrapping insulators, and Case 3: wrapping base frame.

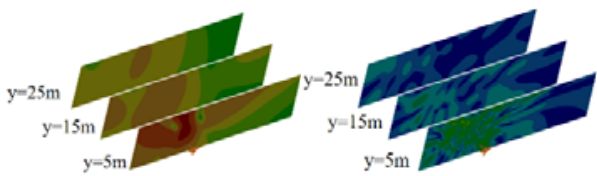

(a) Case $1(200 \mathrm{~Hz} / 1000 \mathrm{~Hz})$

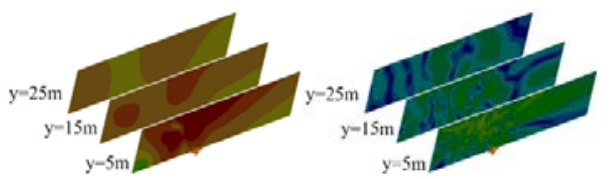

(b) Case $2(200 \mathrm{~Hz} / 1000 \mathrm{~Hz})$

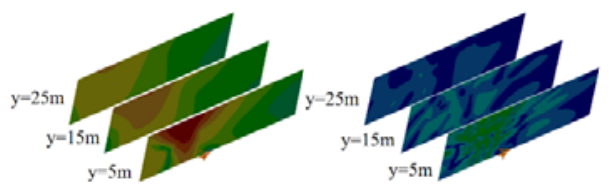

(c) Case $3(200 \mathrm{~Hz} / 1000 \mathrm{~Hz})$

$\begin{array}{llllll}\text { Pressure Amplitude(dB) } & 34 & & 62 & 90 \\ 20 & & 48 & & 76\end{array}$

Fig.16. Spatial distributions of noise on receiving surfaces at $y=5,15$, and $25 \mathrm{~m}$ in three cases

Therefore, the influences on noise are also a comprehensive influence of the whole geometric shape. The far-field noise induced by the pantograph, which is considered a sound source, is a result of the superposition of all components of the pantograph.
Hence, the influence of the shape modification on the far-field noise induced by all components of the pantograph needs to be taken into account. In the next part, the far-field noise characteristics induced by all components of the pantograph body are analyzed.

As shown in Fig. 16, three planes at $y=5,15$, and 25 $\mathrm{m}$ from the center reference point are used to scrutinize the characteristics of the sound propagation in the far-field regions. The three planes show the spatial distribution of noise and they show that at the same plane position, Case 2 provides regions with the highest noise, and Case 3 gives weaker noise than Case 1.

The spectral characteristics of noise induced by the three cases at four monitoring positions i.e. $\mathrm{y}=5,15$, and $25 \mathrm{~m}$ along the lateral distance from the reference center of the pantograph, including $25 \mathrm{~m}$ from the lateral distance from the pantograph reference center and $15 \mathrm{~m}$ downstream along the flow direction, named as $\mathrm{y} 25 \mathrm{~m}-\mathrm{x} 15 \mathrm{~m}$, are analyzed and compared. Numerical results of the comparative analysis are shown in Fig. 17. The results show that when the base frame is wrapped (Case 3), the spectrum of the SPL amplitudes in the far-field region is lower than that of the original case (Case 1). However, at higher frequencies, the difference in the noise spectrum of the SPL amplitudes between the two cases is small. Additionally, the noise amplitudes obtained by the wrapping insulators (Case 2) are higher than the other cases in the whole frequency range for the four monitoring positions. 

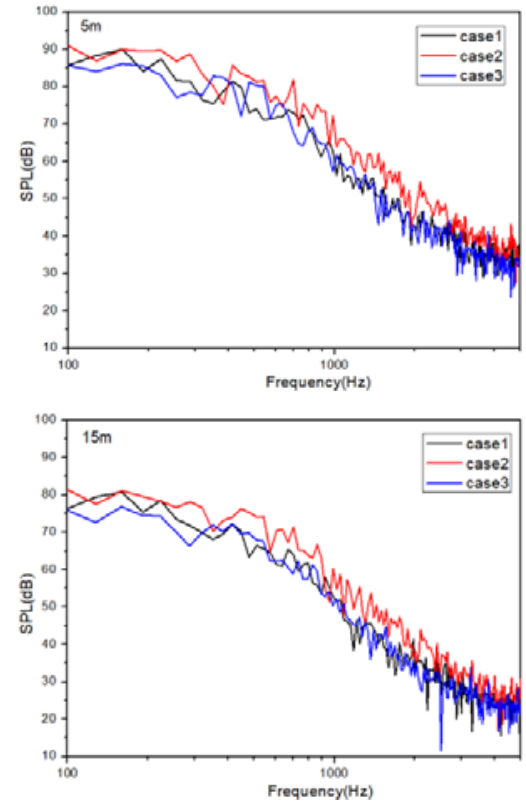
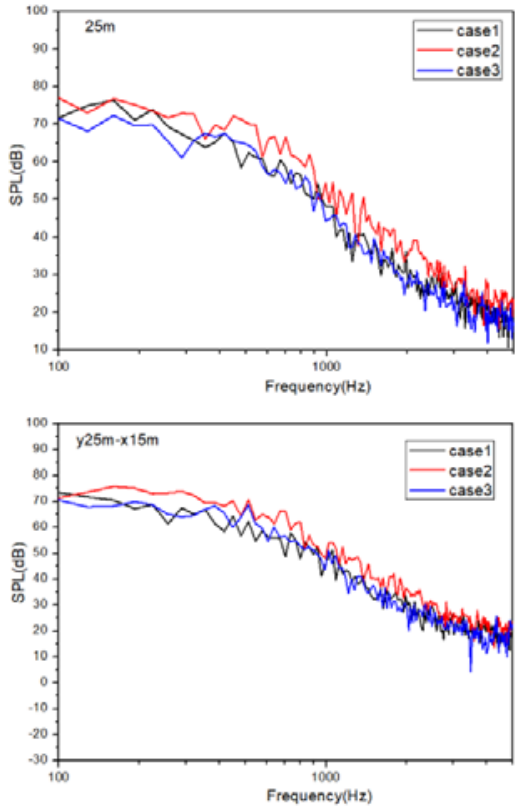

Fig. 17. Noise predictions at far-field monitoring positions $y=5 \mathrm{~m}, 15 \mathrm{~m}$, and $25 \mathrm{~m}$ from center line of pantograph for Case 1: original structure, Case 2: wrapping insulators, and Case 3: wrapping base

frame.

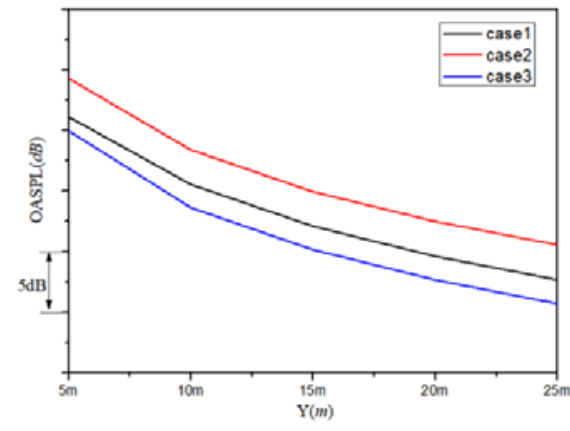

Fig. 18. OASPL at far-field monitoring positions $\mathrm{y}=5,10,15,20$, and $25 \mathrm{~m}$ from center line of pantograph for Case 1: original structure, Case 2: wrapping insulators, and Case 3: wrapping base frame.

To obtain a better understanding of the far-field noise, as seen in Fig. 18, the overall sound pressure level (OASPL) of noise at different monitoring positions in the $y$-direction, which is consistent with the results of spectrum analysis, namely, $y=5,10$, 15,20 , and $25 \mathrm{~m}$. Unsurprisingly, the OASPL decreases with increasing y position for the three cases. Nonetheless, at a fixed monitoring position, the wrapping base frame provides the minimum OASPL, whereas the wrapping insulator gives the maximum noise.

From all the results given in this section, it suggests that for the shape design of the high-speed-train pantograph, if the drag reduction is desired, either the wrapping of the base frame or insulators can be selected. However, the case of the wrapping insulators used in the present work does not play a positive role in noise reduction. Therefore, if the drag reduction and noise reduction are needed simultaneously, the wrapping base frame is a better choice.

\section{CONCLUSION}

This work presents the effects of the structural modification of the pantograph on aerodynamic resistance and noise generated by the pantograph of a high-speed train running at a speed of $300 \mathrm{~km} / \mathrm{h}$ using numerical techniques of IDDES and acoustic FEM. The two designs for the pantograph modification, i.e. the wrapping insulators and wrapping the base frame, are selected. The results obtained by the two modifications are compared with those obtained by the original pantograph (without modification) for the expectation of drag and noise reductions. Through the above analysis and discussion, the numerical results show some interesting phenomena as follows:

(1) With the analysis of the resistance of different components of the pantograph, the most two important parts of the pantograph are the base frame and the insulator. And both them are selected for the modification to achieve the reduction of aerodynamic drag and noise. Compared with the original case, both the modified pantographs are beneficial to reduce the resistance of the pantograph.

(2) In the near field, monitoring probes are set at different positions on the surface of the pantograph. When the insulator is wrapped, most of the noise amplitude of the monitoring probes will be larger than the baseline model. When the base is covered, the noise amplitude at each monitoring point is 
related to the position, but the noise amplitudes at the corner of the upper and lower boom decrease significantly. The noise of surface monitoring probes can reflect the change of unsteady flow field near the object surface. The larger the noise amplitude is, the stronger the sound source intensity will be.

(3) Through the analysis of the far-field noise generated by the three cases, it is found that the modification of the pantograph insulators is beneficial to reduce the aerodynamic resistance, but it is not conducive to noise reduction. However, a positive effect on the drag and noise reductions can be achieved by modifying the base frame of the pantograph

This work can provide sound reference for highspeed-train designers and engineers. However, there is still something worthy of further study. For example, when the insulator is covered, if an elliptical surface is used, the difference in the size ratio of the long and short axis of the covering surface and the shape of it may result in different flow field results; When the base is covered, the size and shape of the covering surface selected for transition between different areas will also have different effects on the results. The adjustment of the above mentioned parameters may have an impact on the design results, and further research on these effects will be carried out in the future work.

\section{ACKNOWLEDGEMENTS}

Author Contributions: Conceptualization, Zhenxu Sun, Guibo Li and Guowei Yang; Data curation, Yongfang Yao and Guibo Li; Formal analysis, Yongfang Yao; Funding acquisition, Zhenxu Sun and Guowei Yang; Investigation, Yongfang Yao and Zhenxu Sun; Methodology, Yongfang Yao; Project administration, Zhenxu Sun and Guowei Yang; Resources, Zhenxu Sun and Guowei Yang; Software, Yongfang Yao; Supervision, Zhenxu Sun and Guowei Yang; Validation, Yongfang Yao, Guijun Chen and Zhenxu Sun; Visualization, Yongfang Yao; Writing-original draft, Yongfang Yao; and Writing-review and editing, Prasert Prapamonthon ,and Zhenxu Sun.

This work is supported by the Strategic Priority Research Program of the Chinese Academy of Sciences (class B) (Grant No. XDB22020000), the Informatization Plan of the Chinese Academy of Sciences (Grant XXH13506-204), and Youth Innovation Promotion Association CAS(2019020). Also, the Computing Facility for the 'Era' petascale supercomputer of Computer Network Information Center of the Chinese Academy of Sciences is gratefully acknowledged.

\section{REFERENCES}

Gritskevich, M. S., A. V. Garbaruk, J. Schütze and F. R. Menter (2012). Development of DDES and IDDES formulations for the $\mathrm{k}-\omega$ shear stress transport model. Flow, Turbulence and Combustion 88(3), 431-449.
Guang, Y., G. H. Peng (2019). Methods of reducing aerodynamic noise of pantograph. Foreign railway vehicles 6(2), 39-42. (In Chinese)

Huang, K. L., T. C. Yuan, J. Yang and R. G. Song (2019). Aerodynamic Noise Analysis and Noise Reduction Study of Pantograph of High-speed Train. Computer Measurement \& Control 27(3), 192-196. (In Chinese).

Ikeda, M., M. Suzuki and K. Yoshida (2006). Study on optimization of panhead shape possessing low noise and stable aerodynamic characteristics Quarterly. Report of Railway Technical Research Institute 47(2), 72-77.

King, W. F. (1996). A precise of development in the aeroacoustics of tast trains. Journal of Sound and Vibration 193(1), 349-358.

Liu, H. T. and Z. L. Xu (2018). Study on Drag and Noise Reduction of Pantograph Rods based on Bionic Non-smooth Structures. Noise and Vibration Control (38), 269-272.

Liu, J. L., M. G. Yu and A. Q. Tian (2018). Study on the aerodynamic noise characteristics of the pantograph of the high-speed train. Journal of Mechanical Engineering 54.

Lockard, D. P. (2011). Summary of the tandem cylinder solutions from the benchmark problems for airframe noise computations // Workshop 49th AIAA Aerospace Sciences Meeting including the New Horizons Forum and Aerospace Exposition. Orlando, 1-22.

Masson, E., N. Paradot and E. Allain (2012). The numerical prediction of the aerodynamic noise of the TGV POS high-speed train power car. Noise and vibration Mitigation for Rail Transportation Systems, Notes on Numerical Fluid Mechanics and Multidisciplinary Design, Springer Berlin Heidelberg, 437-444.

Menter, F. R. (1994). Two-equation eddy-viscosity turbulence models for engineering applications. AIAA 32(8), 1598-1605.

Neuhart, D., L. Jenkins, M. Choudhari and M. R. Khorrami (2009). Measurements of the Flowfield Interaction between Tandem Cylinders. 15th AIAA /CEAS Aeroacoustics Conference (30th AIAA Aeroacoustics Conference), 1-18.

Noger, C., J. C. Patrat, J. Peube and J. L. Peube (2000). Aeroacoustical study of the TGV pantograph recess. Journal of Sound and Vibration 231(3), 563-575.

Shi, R. C. (2018) Low noise design of high speed train pantograph based on rod section Optimization. Master Degree Thesis, Southwest Jiaotong University, (In Chinese).

Shur, M. L., P. R. Spalart, M. K. Strelets and A. K. Travin (2008). A hybrid RANS-LES approach with delayed-DES and wall-modelled LES capabilities. International Journal of Heat and Fluid Flow 29(6), 1638-1649. 
Spalart, P. R., S. Deck, M. L. Shur K. D. Squires, M. K. Strelets and A. Travin (2006). A new version of detached-eddy simulation, resistant to ambiguous grid densities. Theoretical and Computational Fluid Dynamics 20(3), 181195.

Spalart, P. R. (1997). Comments on the feasibility of LES for wings, and on a hybrid RANS/LES approach. In: Advances in DNS/LES, Greyden Press, Columbus, OH, USA.

Sun, X. Q. and H. Xiao (2018). Numerical modeling and investigation on aerodynamic noise characteristics of pantographs in high-speed trains. Complexity, 1-12.

Sun, Z. X., D. L. Guo, S. B. Yao and G. W. Yang (2013). Identification and suppression of noise sources around high speed trains. Engineering Applications of Computational Fluid Mechanics 7(1), 131-143.

Suzuki, M., M. Ikeda and K. Yoshida (2008). Study on numerical optimization of cross-sectional panhead shape for high-speed train. Journal of Mechanical Systems for Transportation and Logistics 1(1), 100-110.

Takaishi, T. and C. M. Kato (2004). Method of evaluating dipole sound source in a finite computational domain. Nihon Kikai Gakkai Ronbunshu, B Hen/Transactions of the Japan Society of Mechanical Engineers, Part B, 70(698), 2523-2530.

Takeshi, M. (2016). A Basic Research on Aerodynamic Noise Reduction Techniques for a Pantograph Head Using Plasma Actuators. Foreign railway vehicles 53 (2), 29-33.

Takeshi, S. and X. L. He (2010). Aerodynamic noise reduction using porous materials and their application to high speed pantographs Foreign Locomotive \& Rolling Stock Technology, (005), 23-27.
Tian, H. Q. (2007). Train aerodynamics. Beijing: China Railway Publishing Press, 160-161.

Wang, Y. Y., J. S. Zhou, D. Gong and H. Liu (2018). Study on bionic noise reduction and aerodynamic noise distribution characteristics for high-speed train's pantographs. Noise and vibration control (38), 348-352 (In Chinese).

Xu, Z. L., H. T. Liu, C. W. Wang and Y. H. Chen (2020). Study on Drag and Noise Reduction of Pantograph Rods in High Speed Train. Journal of East China Jiaotong University (37), 1-6.

Yao, S. B., D. L. Guo, G. W. Yang and M. G. Li (2012). Distribution of high-speed trains aerodynamic drag. Journal of the China railways 34 (7), 18-23.

Yao, Y. F., Z. X. Sun, W. Liu and P. Prapamonthon (2019). Analysis of Aerodynamic Noise Characteristics of Pantograph in High Speed Train. Acta Scientiarum Naturalium universitatis Pekinensis 56, 385-398. (In Chinese)

Yu, H. H., J. C. Li and H. Q. Zhang (2013). On aerodynamic noises radiated by the pantograph system of high speed trains. Acta Mechanica Sinica 29 (3), 399 - 410.

Zhang, S.G. (2009). Noise mechanism, sound source identification and control of high-speed train in the speed of $350 \mathrm{~km} \cdot \mathrm{h}-1$. Zhongguo Tiedao Kexue/China Railway ence 30, 86-90. (In Chinese)

Zhang, Y.D., J.Y. Zhang, T. Li and L. Zhang (2017). Investigation of the aeroacoustic behavior and aerodynamic noise of a high-speed train pantograph. Science China Technological Sciences 60,561-575. 